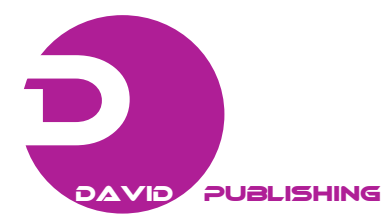

\title{
Dual Active Bridge Converter as Gyrator
}

\author{
Walbermark M. dos Santos and Denizar C. Martins \\ INEP, Power Electronics Institute, Federal University of Santa Catarina, Florianópolis (SC) 88040-970, Brazil
}

Received: August 14, 2013 / Accepted: September 17, 2013 / Published: February 28, 2014.

\begin{abstract}
This work presents the design and control of the DAB (dual active bridge) converter employing gyrator's theory. A brief summary of the theory is introduced, and its application for the DAB converter is verified with a design example. After that, it develops the control and show results of simulations and experiment. Though the commutation not is studied, the advantage of method is the easy way for project and control of the DAB converter.
\end{abstract}

Key words: DAB converter, gyrostatic coefficient, gyrator theory, POPI circuit.

\section{Introduction}

DAB (dual active bridge) converter [1, 2] are topologies formed by two full bridge converters in the configuration back-to-back, interconnected by a high frequency inductive AC link, which can be a transformer (Fig. 1) or a single inductor.

In the technical nomenclature, the DAB converter is called a two-port converter. Both ports may be connected to the voltage sources, or one port (main port) has a voltage source connected to the input, with the other port feeding a resistive load.

Particularly, in the configuration where port 2 feeds a load with resistive behavior, the values of the transmission inductance and capacitor filter become the first elements to be calculated. The design of these should consider the operating angle, the maximum power transferred, the value of the supplied load, the output voltage, the current peaks acceptable in the switch, etc..

Since the project constraints are met, several methodologies can be applied to the design of these elements. This paper presents a simple design methodology, based on the representation of the

Corresponding author: Denizar C. Martins, professor, research fields: power electronics, dc-ac converter and power quality. E-mail: denizar@inep.ufsc.br. converter DAB as a gyrator.

The gyrator concept was introduced in literature by Tellegen [3], in the $40 \mathrm{~s}$. According to this reference, there are basically five types of linear elements used in electric circuits: resistors, capacitors and inductors, all these elements with one port; the transformer and the fifth element, defined as gyrator with two ports.

The gyrator is an element that can be viewed as a variation of the transformer [4]. It is a realizable two-port network, nonreciprocal, lossless and storage less, which couples the input port to output port through a coefficient called the gyrostatic coefficient [5].

One of the basic requirements of the system to be represented by a gyrator is to satisfy the condition of conservation of power or POPI condition (power output equals power input). Several switched power converter can meet the condition POPI through a closed loop control of voltage or current. However, the $\mathrm{DAB}$ converter is considered a natural gyrator, without any loop control [5].

In this way, the proposed methodology is based on the fact that the DAB converter is a natural gyrator. It should be emphasized that the theory of the gyrator, can represent the $\mathrm{DAB}$ converter in a more compact form, without losing resolution in the reproduction of its behavior in steady state and transient operation.

The equivalent circuit of the DAB converter using of 
the gyrator theory, can be used for calculating the inductance transmission and filter capacitor of the load. The design does not consider the current peaks that will be transmitted, and then the general theory of the DAB converter is responsible to do in this study. However, it can be quickly realizable by a simple methodology, applying only a few equations.

The proposed methodology is presented as follows: Section 2 introduces the basic mathematical relationships of the gyrator and the DAB converter. The proposed design methodology is presented in Section 3. Section 4 presents the simulations and experimental results, and finally, Section 5 presents the conclusions of this work.

\section{The Gyrator and the DAB Converter}

This section presents a brief review of the gyrator's theory, followed by its application in the DAB converter design.

\subsection{The Gyrator}

As an ideal transformer, the gyrator has no losses or accumulate energy, as part of a wide variety of processing circuits of energy called POPI [6]. As has been said earlier, the gyrator has two ports, and such as the transformer, any component connected to the port of a gyrator can be reflected to the other port.

But during this reflection one realizes the main feature of the gyrator. Contrary to the transformer, that reflects the element of one "port" to another just by changing its value and keeping their "nature", the gyrator keeps a peculiar characteristic: It can convert ("rotate") an element, when reflecting from one port to another in its dual, for example: In one of the "port" of the gyrator is connected a voltage source which, when reflected to another port, it will appear as the current source and vice-versa.

Thus, the voltage-current, current-voltage, capacitance-inductance, inductance-capacitance, resistance-conductance and conductance-resistance conversion can easily be done in a circuit using the gyrator [6-8]. These transformations may lead to a simplification of complex circuits, improving analysis and understanding of their behavior in certain condition.

The symbology of the gyrator is shown in Fig. 2 [3, 6, 8], which highlights the two basic types of gyrators: gyrator conductance $g$, with dimension $1 / \Omega$, and gyrator resistance $r$, with dimension $\Omega$.

In the Fig. 2a, the relationships between voltage and current of the ports are given by:

$$
\left\{\begin{array}{l}
i_{1}= \pm g \cdot v_{2} \\
i_{2}=\mp g \cdot v_{1}
\end{array}\right.
$$

and the set of equations for the gyrator resistance in Fig. $2 b$ is:

$$
\left\{\begin{array}{l}
v_{1}= \pm r \cdot i_{2} \\
v_{2}=\mp r \cdot i_{1}
\end{array}\right.
$$

Unlike a transformer, in which the output current depends on input current, for example, in a gyrator the output current depends on the input voltage, as seen in Eqs. (1) and (2).

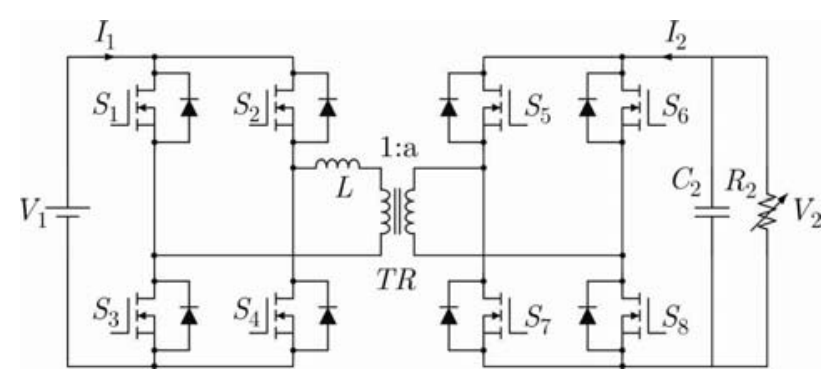

Fig. 1 DAB converter.

(a)

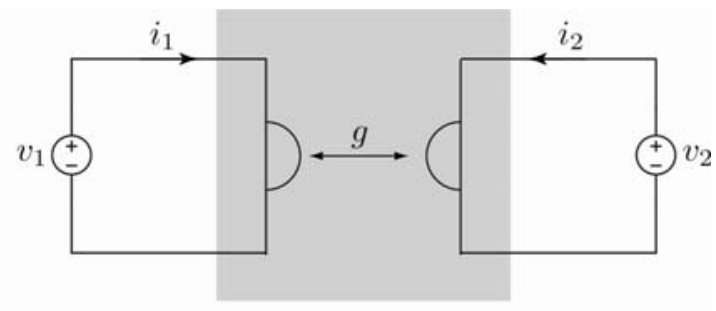

(b)

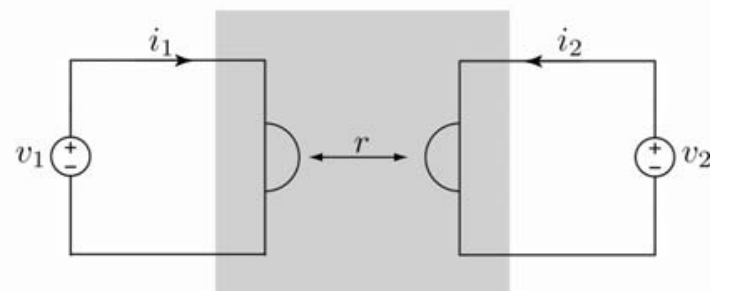

Fig. 2 Tellegen's gyrator symbols: (a) gyrator conductance; (b) gyrator resistance. 
The Eq. (1), which defines the output current $i_{2}$, can be represented by the electrical circuit shown in Fig. 3. Thus, the gyrator conductance is employed to build an electrical circuit which reproduces such equation. In this situation, the input source $v_{l}$ reflected (multiplied by the gyrator conductance) to the port 2 (output port) "becomes" a current source with value equal to the output current $i_{2}$.

The power delivered to the impedance $Z_{2}$ is found with:

$$
p_{2}=i_{2} \cdot v_{2}=g \cdot v_{1} \cdot v_{2}=\left(g \cdot v_{1}\right)^{2} Z_{2}=\frac{\left(v_{2}\right)^{2}}{Z_{2}}
$$

As the system is conservative, the output power is equal to the input power. Thus, the power generated by the source $v_{1}$ is consumed by the impedance $Z_{2}$, as defined by:

$$
p_{1}=p_{2} \Leftrightarrow i_{1} \cdot v_{1}=i_{2} \cdot v_{2}
$$

From Eqs. (1), (2) and (4), the power $p$ transmitted between the ports of the gyrator can be determined by any equations of:

$$
\left\{\begin{array}{l}
p=v_{1} \cdot v_{2} \cdot g \\
p=i_{1} \cdot i_{2} \cdot r \\
p=\frac{i_{1} \cdot i_{2}}{g} \\
p=\frac{v_{1} \cdot v_{2}}{r}
\end{array}\right.
$$

The set of Eq. (5) describes the instantaneous power transmitted through the gyrator. If the physical system studied is a switched converter, these equations describe the behavior of the instantaneous average power over a period of switching of these converters [5].

\subsection{The DAB Converter}

The DAB converter is presented in Fig. 1. It is seen that port 1 is fed by a DC voltage source $V_{l}$ and port 2 feeds a resistive load $R_{2}$ in parallel with a capacitive filter $C_{2}$.

Neglecting the losses, the power transmitted between ports is given by Eq. (6) $[1,2]$.

$$
P=\frac{V_{1} \cdot V_{2}}{a \cdot \omega \cdot L} \varphi\left(1-\frac{|\varphi|}{\pi}\right)
$$

where,

$V_{1}, V_{2}$ are the voltages at ports 1 and 2, respectively;

$a=\frac{N_{1}}{N_{2}}$ is the transformer turns ratio;

$\varphi$ is angle between the voltages at the ports;

$L$ is total series inductance;

$\omega=2 \pi f$ and $f$ is converter operating frequency.

Based on Eq. (6), and considering the conservative system, the output current of the converter is found with:

$$
\frac{P}{V_{2}}=I_{2}=\frac{V_{1}}{a \cdot \omega \cdot L} \varphi\left(1-\frac{|\varphi|}{\pi}\right)
$$

As seen in Eq. (7), the output current of the DAB converter is the product of input voltage by a variable that has dimension of conductance. Thus, the current in the load can be redefined as:

$$
I_{2}=g \cdot V_{1}
$$

With:

$$
g=\frac{1}{a \cdot \omega \cdot L} \varphi\left(1-\frac{|\varphi|}{\pi}\right)
$$

Comparing Eq. (8) with Eq. (1) is easy to see that the element $g$ is the gyrator conductance of the converter DAB.

Based on Eq. (8), we can present an electrical circuit of the load port, as shown in Fig. 4. Thus, the power demanded by the load resistance $R_{2}$ is represented by:

$$
P_{2}=g \cdot V_{1} \cdot V_{2}=I_{2} \cdot V_{2}=\frac{\left(V_{2}\right)^{2}}{R_{2}}=\left(g \cdot V_{1}\right)^{2} \cdot R_{2}
$$

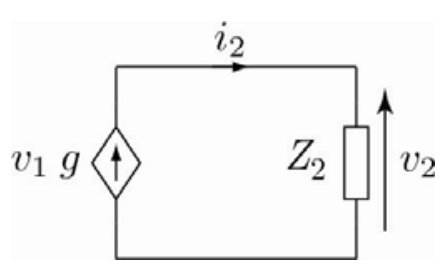

Fig. 3 Equivalent electrical circuit of the port 2.

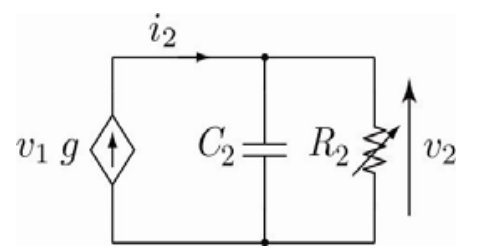

Fig. 4 Equivalent electrical circuit of the load port 2. 
It is seen that, according to the gyrators theory, the load sees the voltage source that feeds the port 1 as a current source reflected to port 2. The circuit shown in Fig. 4, although simple, can represent with good fidelity the behavior in steady state and transient response of the DAB converter.

From Eq. (9) we can write:

$$
x=\varphi\left(1-\frac{|\varphi|}{\pi}\right)
$$

Where:

$$
x=g \cdot a \cdot \omega \cdot L
$$

The graph shown in Fig. 5 is extremely important for the proposed methodology, because through it that set up directly the angle of operation of the converter and the variable $x$ which is proportional to the ratio of transformation, the gyrostatic coefficient value, the switching frequency and inductance of transmission. The variable $x$ can be interpreted as the per unit current or power, depending on the angle between the ports of the gyrator.

\section{Proposed Methodology}

Considering the main specifications of the system: the power $P$, the input and output voltages $\left(V_{1}\right.$ and $\left.V_{2}\right)$, transformer turns ratio $a$ and the operating frequency of the converter $f_{s}$, from Eq. (7) the gyrator conductance of the DAB converter that promotes the desired power transfer is easily calculated as the ratio between the power and the product of voltages, as:

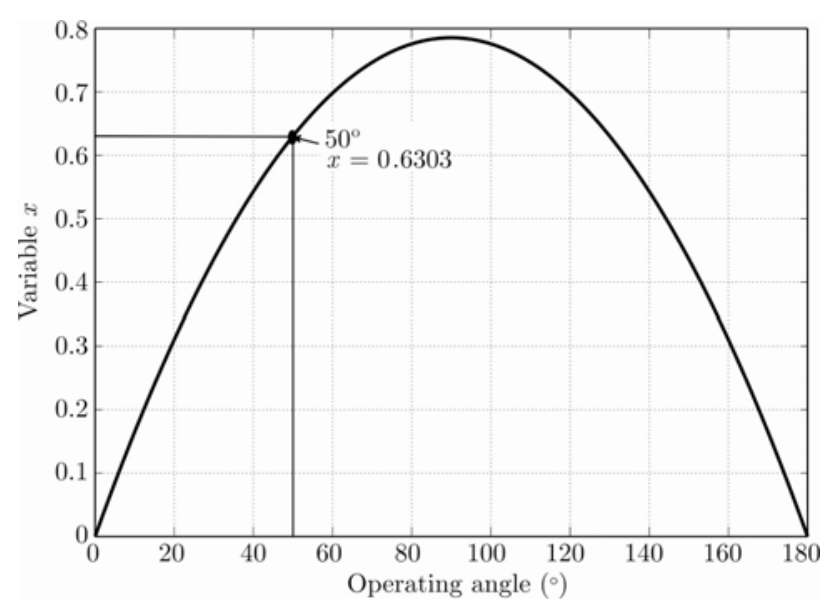

Fig. 5 Variation of the variable $x$ as a function of the converter operating angle.

$$
g=\frac{P}{V_{1} \cdot V_{2}}
$$

The variable $x$ is chosen in Fig. 5 according to the desired power. Thus, the inductance of transmission is determined with:

$$
L=\frac{x}{g \cdot a \cdot \omega}
$$

and the value of the equivalent load resistance is given by:

$$
R_{2}=\frac{P_{2}}{\left(g \cdot V_{1}\right)^{2}}
$$

The calculation of the capacitor is realized considering that the variations of the energy in itself is given by:

$$
E=\frac{C_{2} \cdot\left(V_{\max }^{2}-V_{\min }^{2}\right)}{2}
$$

where, $E$ is the energy accumulated in the capacitor; $C_{2}$ is the capacitance and $V_{\max }$ and $V_{\min }$ are the maximum and minimum voltages over the output capacitor, respectively.

Since the energy $E$ is:

$$
E=p \cdot t
$$

from Eqs. (16) and (17), the capacitance $C_{2}$ is obtained with:

$$
C_{2}=\frac{2 P}{\left(V_{\max }^{2}-V_{\min }^{2}\right)^{2}} t
$$

Alternatively, from Eqs. (15) and (18) the capacitance $C_{2}$ is:

$$
C_{2}=\frac{2 \cdot\left(g \cdot V_{1}\right)^{2} R_{2}}{V_{\max }^{2}-V_{\min }^{2}} t
$$

From Eq. (19), the variable $t$ is the period that the capacitor will maintain the load voltage under the specified power. The power source $V_{l}$ delivers power to source $V_{2}$ only in the time interval corresponding to the angle $\varphi$, whereas in the remaining period the capacitor will supply power to the load. The time interval $t$ is determined by:

$$
t=\left(\frac{2 \pi}{\omega}-\frac{\varphi}{\omega}\right)
$$

where, $2 \pi / \omega$ is the time interval corresponding to the operating period of the converter. The term $\varphi / \omega$ is 
the time interval where the port 1 delivers power to the port 2 .

From Eqs. (20) and (19), the capacitance $C_{2}$ is found with:

$$
C_{2}=2 \cdot\left(g \cdot V_{1}\right)^{2} \cdot R_{2}\left(\frac{2 \pi-\varphi}{2 \pi f_{s}}\right) \cdot \frac{1}{\left(V_{\max }^{2}-V_{\min }^{2}\right)}
$$

Notice that the converter can operate under zero angle, but the capacitor still should maintain the voltage in the load. Thus $C_{2}$ is finally obtained from Eq. (22):

$$
C_{2}=\frac{2 \cdot\left(g \cdot V_{1}\right)^{2} \cdot R_{2}}{f_{s}} \cdot \frac{1}{\left(V_{\max }^{2}-V_{\min }^{2}\right)}
$$

Eq. (22) is employed to determine the output capacitor of the DAB in a quite conservative way. It is observed that it takes into account the coefficient gyrostatic of the converter, the operating frequency and the value of load resistance. Summarizing, the proposed methodology for the design of the main elements of the DAB converter employs the Fig. 5 and the Eqs. (13)-(15) and (22).

\section{Simulation and Experimental Results without Control}

To validate the proposed methodology, a DAB converter with the specifications given in Table 1 is designed.

According to the desired voltage and power, the gyrostatic coefficient is determined from Eq. (13) as $g$ $=0.0629 \Omega$. With the desired operating angle inserted in Fig. 5, we can find $x=0.6303$. Substituting $g$ and $x$ in Eq. (14), we obtained $L=31.89 \mu \mathrm{H}$. From Eq. (15), the equivalent load resistance is $R_{2}=13.46 \Omega$. Finally, add up all the necessary data in Eq. (24) the capacitance of output filter results $C_{2}=7.15 \mu \mathrm{F}$.

For building and testing a prototype, the element values used were: $L=33 \mu \mathrm{H}, R_{2}=15.14 \Omega$ and $C_{2}=47 \mu \mathrm{F}$. The capacitor used is very larger that is calculated. This change is justified by the RMS current of the capacitor.

Fig. 6 shows the circuit DAB converter built. To make the comparison between theoretical and experimental results, includes some parameters such as: impedance values of the cables between the source
Table 1 Main specifications.

\begin{tabular}{ll}
\hline Parameter & Value \\
\hline Voltage at port 1 & $V_{l}=130 \mathrm{~V}$ \\
Voltage at port 2 & $V_{2}=110 \mathrm{~V}$ \\
Total power & $P=900 \mathrm{~W}$ \\
Switching frequency & $f_{s}=50 \mathrm{kHz}$ \\
Maximum voltage & $V_{\max }=120 \mathrm{~V}$ \\
Minimum voltage & $V_{\min }=100 \mathrm{~V}$ \\
Desired operation angle & $\varphi_{o}=50^{\circ}$ \\
Transformer turn ratio & $a=1$ \\
\hline
\end{tabular}

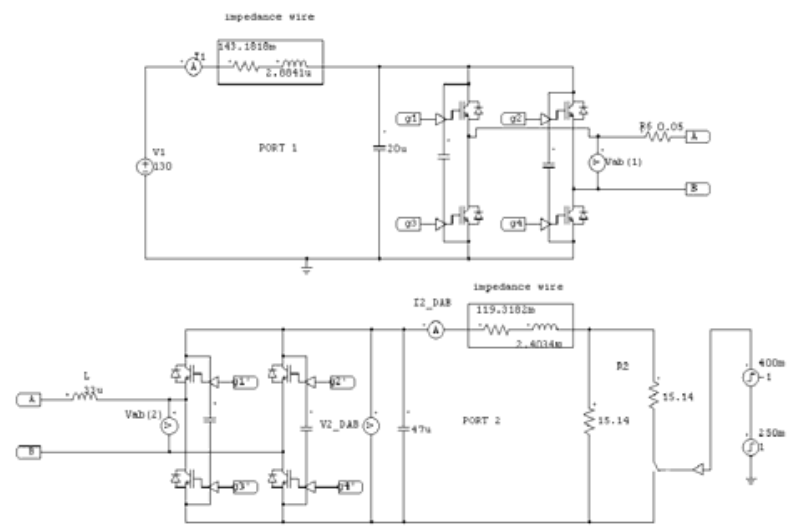

Fig. 6 Simulated DAB converter.

and a port 1; impedance values of the cables between port 2 and the load; resistance value of inductor and clamping capacitors that are attached across of the switch (with the objective to mitigate the voltage peaks in the switch due to parasitic inductances).

This section is divided in two subsections: The first ones are compared with the simulation results between the DAB converter and equivalent gyrator circuit. After that, we compare simulation and experimental results of the DAB converter.

\subsection{Comparing DAB and Dyrator}

Fig. 7 shows the equivalent gyrator circuit of the DAB converter. To compare the circuits, was applied step load in the port 2 at $t=250 \mathrm{~ms}$. This instant, the resistance change of $R_{2}=13.46 \Omega$ for $R_{2}=6.73 \Omega$. At $t$ $=400 \mathrm{~ms}$, the resistance returns to $R_{2}=13.46 \Omega$.

Fig. 8 shows the behavior of the voltage and load current for both circuits. Note that, looking from the port 2, the DAB converter behaves as current source, because without any control loop, the current in the 


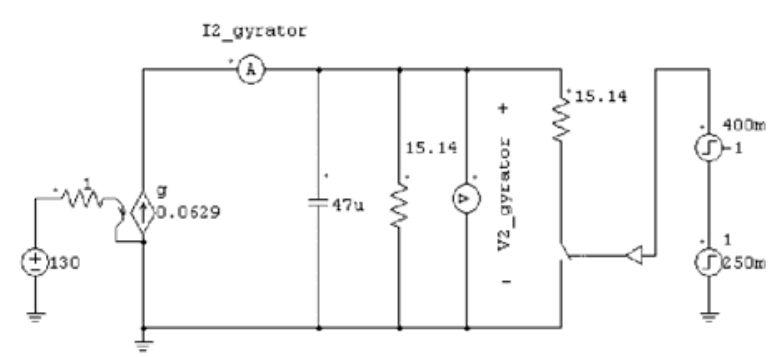

Fig. 7 DAB converter in the form of gyrator.
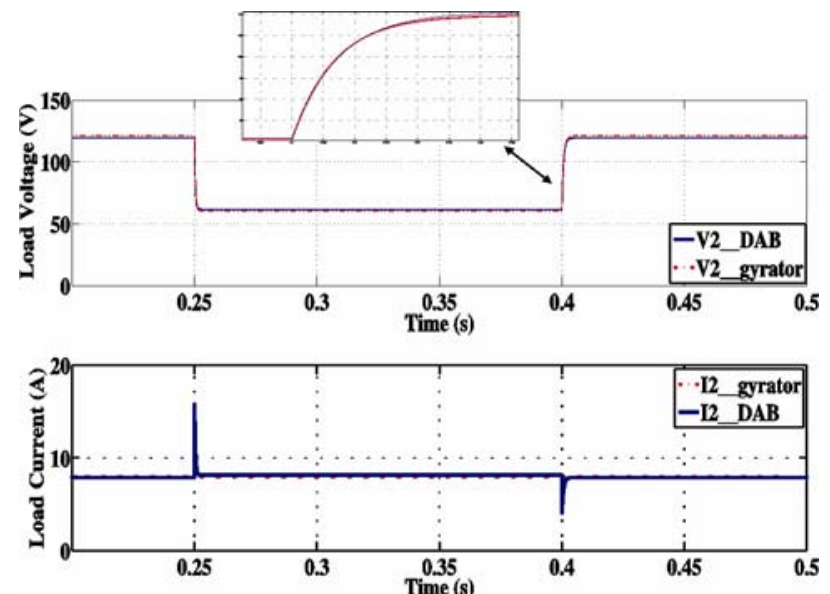

Fig. 8 Comparing (Figs. 6 and 7) voltage and load current in DAB and gyrator circuit.

load tends to return to initial value after the transients. The load output voltage decreases, and the power delivery from port 1 . The voltage dynamic behavior is evidenced (in evidence the dynamic output of the load), where it is found that the response of both circuits is almost identical.

As seen in Fig. 8, the circuit modeling from the gyrator does not reproduce the transient current, a fact that was expected. Therefore, this circuit is not suitable to study this phenomenon. However, as reported in the literature, the DAB circuit, obtained from the representation as gyrator, reproduces quite closely some aspects of the behavior of this converter, and can be useful, for example, to obtain the voltage control loop.

\subsection{Comparison between Simulation and Experimental} Results of the DAB Converter

From Figs. 9-11, we have the results obtained from simulations and, from Figs. 12-14 the results are obtained from experimental tests of the DAB converter. The variables correspond to Fig. 6 , where $I_{2}$ equals to
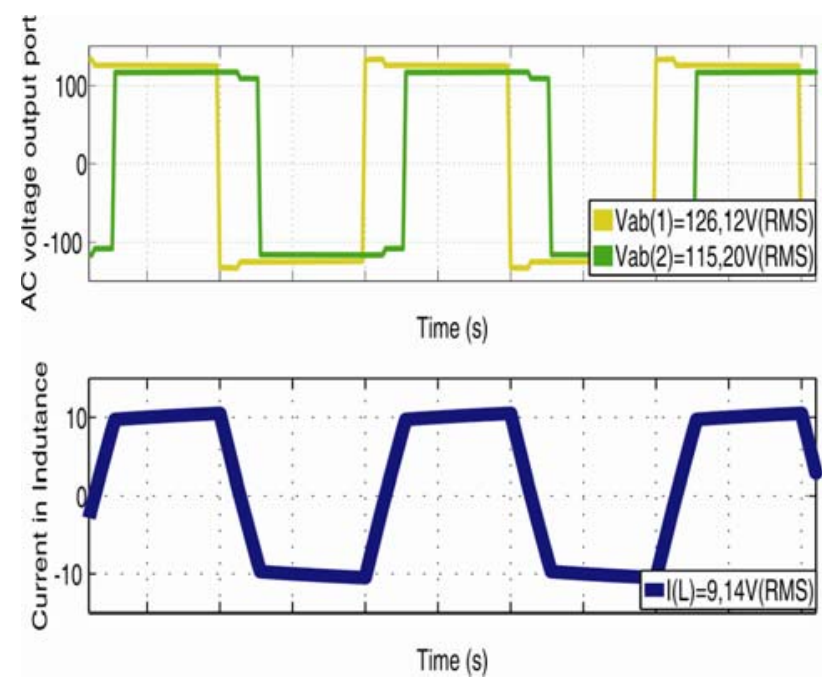

Fig. 9 Simulation AC voltage $\operatorname{Vab}(1)$ and $\operatorname{Vab}(2)$ in Fig. 6.

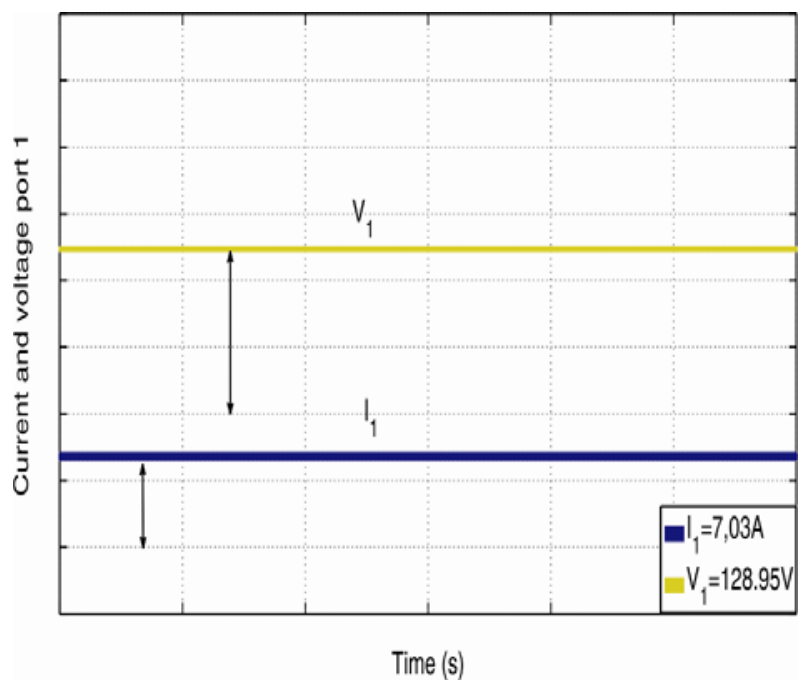

Fig. 10 Simulation of the input current $(5 \mathrm{~A} / \mathrm{div})$ and voltage ( $50 \mathrm{~V} / \mathrm{div})$ port 1.

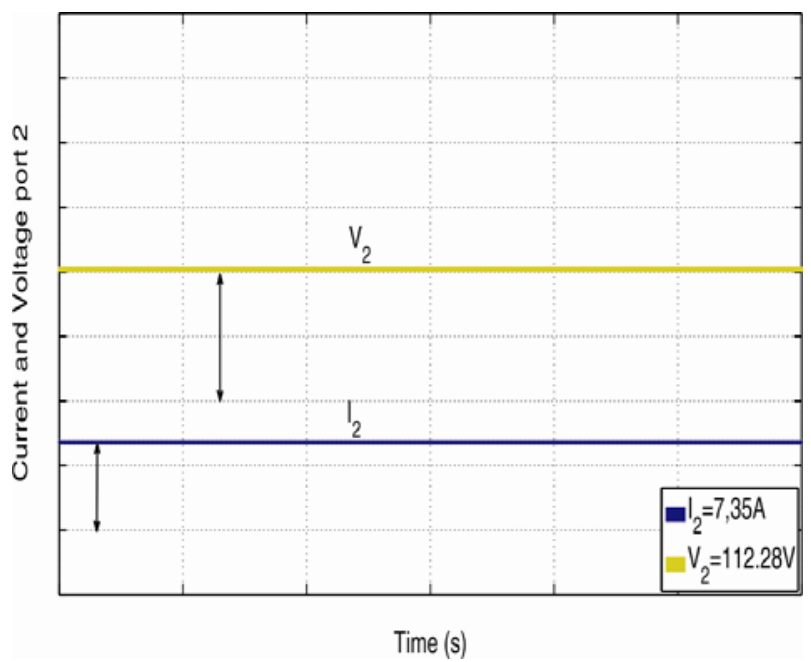

Fig. 11 Simulation of the output current $(5 \mathrm{~A} / \mathrm{div})$ and voltage (50 V/div) port 2. 


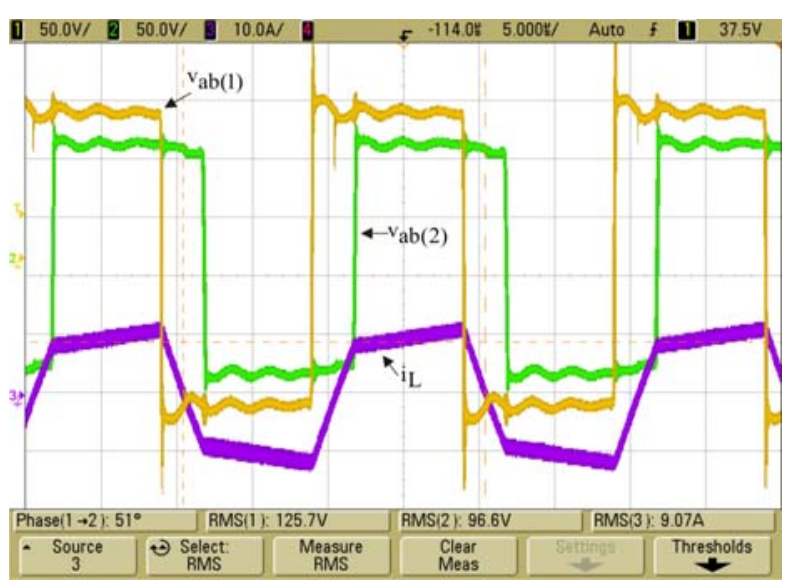

Fig. 12 Experimental AC voltage $\operatorname{Vab}(1)$ and $\operatorname{Vab}(2)$ and inductor current in Fig. 6.

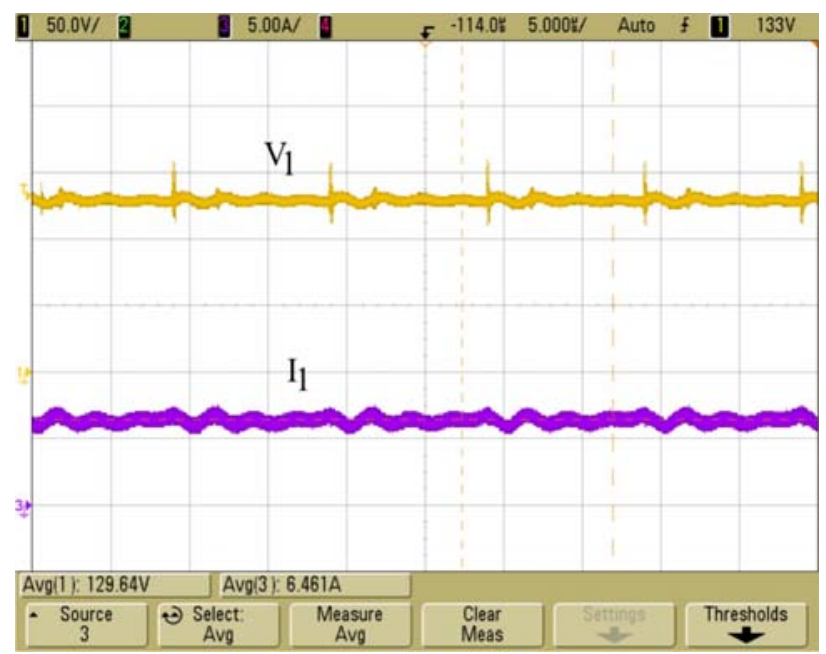

Fig. 13 Experimental input current $(5 \mathrm{~A} / \mathrm{div})$ and voltage (50 V/div) in port 1.

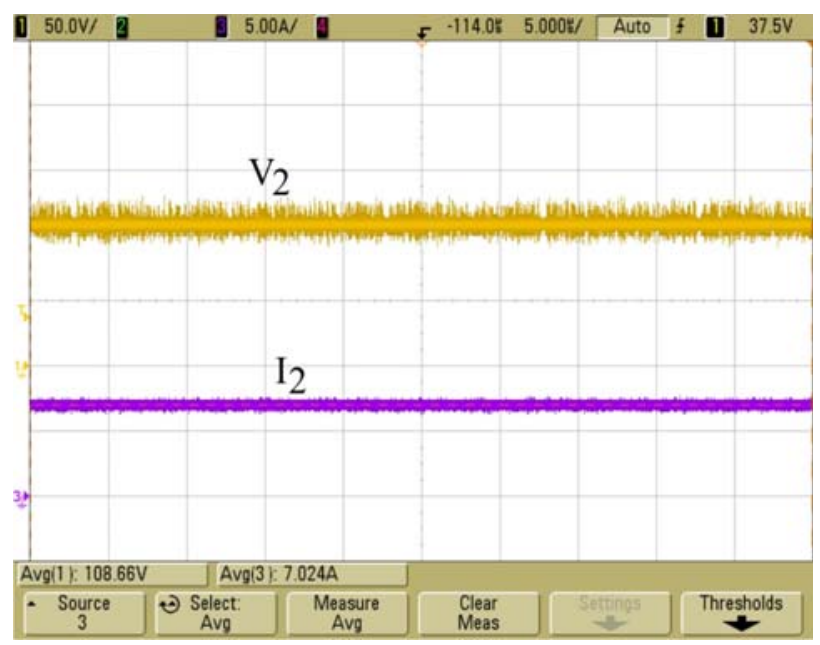

Fig. 14 Experimental output current (5A/div) and output voltage (50 V/div) in port 2.

$I_{2} D A B, V_{2}$ equals to $V_{2} D A B$. The voltage $V_{1}$ is measured in the input of the port 1 , after the voltage drop on the cable.

Comparing the results obtained in both cases, it is observed that the waveforms are consistent both in shape and value. Observing the experimental waveforms, there is a power that was extracted about the $837.60 \mathrm{~W}$ from port 1 , and it is delivered approximately $763.22 \mathrm{~W}$ to the load. At this point the converter would then operate with a yield of around $91.11 \%$. In computer simulation, using these values of voltage and load, it was in a theoretical power transmission around 825.25 W.

These and other discrepancies in values certainly are due to some factors that are not included in the circuit simulation, for example: noise by switching, parasitic elements of the system (capacitance, inductance, resistance variation with frequency), inserted errors by measurement, true characteristics of the components, etc..

Despite the difference between simulation and experimental results, it can be seen that the methodology is feasible to be applied. As the gyrator is a linear circuit, possibly for a lower operating angle, the response of DAB and its equivalent gyrator circuit become more similar.

\section{Simulation and Experimental Results with Control}

The development of control was based on Fig. 15. The transfer function around at operation point is:

$$
\Delta V_{2}=\frac{R_{2}}{C_{2} R_{2} s+1} \Delta I_{2}
$$

Where:

$$
\Delta I_{2}=\Delta g \cdot V_{1}
$$

Considering the approach:

$$
\varphi\left(1-\frac{|\varphi|}{\pi}\right) \approx \frac{8 \sin (\varphi)}{\pi}
$$

the change of gyrator around operation point is given by:

$$
\Delta g=\frac{8}{a \cdot \omega \cdot L \cdot \pi^{2}} \Delta \varphi
$$

The control voltage $V_{2}$ is made as show in Fig. 16 . 


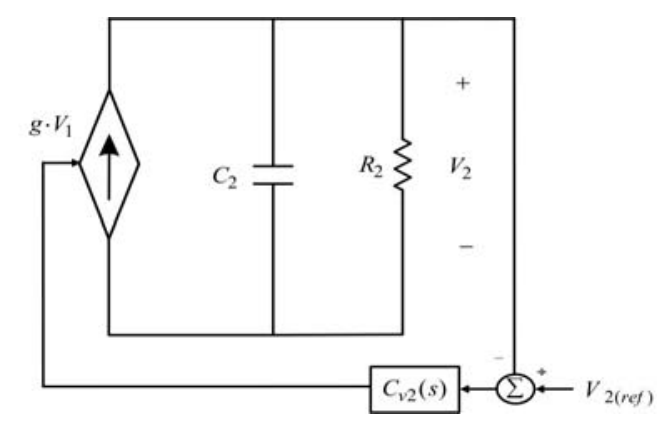

Fig. 15 Control voltage $V_{2}$.

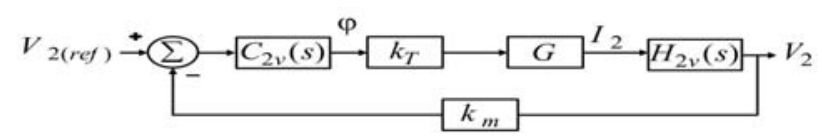

Fig. 16 Loop control voltage $V_{2}$.

where,

$k_{m}$ is gain voltage sensor;

$k_{T}$ is gain phase shift DSP;

$G=8 V_{l} / a \omega L \pi^{2}$ is gain loop control;

$\mathrm{H}_{2}(\mathrm{~s})=\mathrm{R}_{2} /\left(\mathrm{R}_{2} \mathrm{C}_{2} \mathrm{~S}+1\right)$ is the transfer function voltage.

Fig. 17 shows the frequency response transfer function compensated open-loop of voltage. The compensator used is PI, and cutoff frequency was around at $200 \mathrm{~Hz}$. Looking at the phase margin is at around $50 \mathrm{~Hz}$, which ensures its stability.

Figs. 18 and 19 respectively show the simulation response of the voltage and power for a change load. Look the voltage is regulated at 110 V. Fig. 20 shows the experimental result of the voltage and current for a change load. Fig. 21 shows the experimental power consumed by load.

\section{Conclusions}

This paper has presented a methodology for the design and control of the DAB converter applying the theory of gyrator. It was shown that the use of simple equations derived from the representation of the converter as a gyrator can be used to determine the capacitance and inductance of the converter.

The methodology was applied in the design and control of a converter that was simulated and satisfactory response. Experimental results are presented, demonstrating satisfactory response of the method in practice.

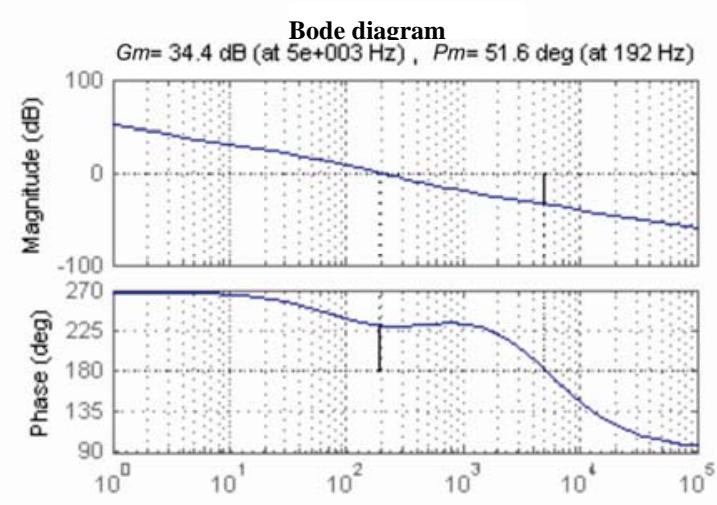

Fig. 17 Frequency response for loop control.

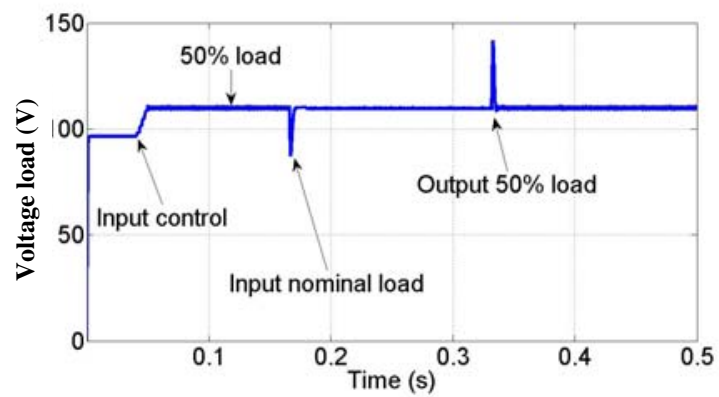

Fig. 18 Simulation of the response voltage for change load.

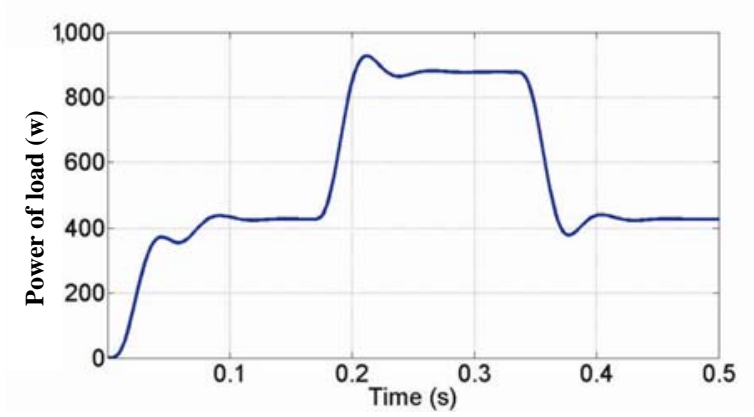

Fig. 19 Simulation of power consumed by load.

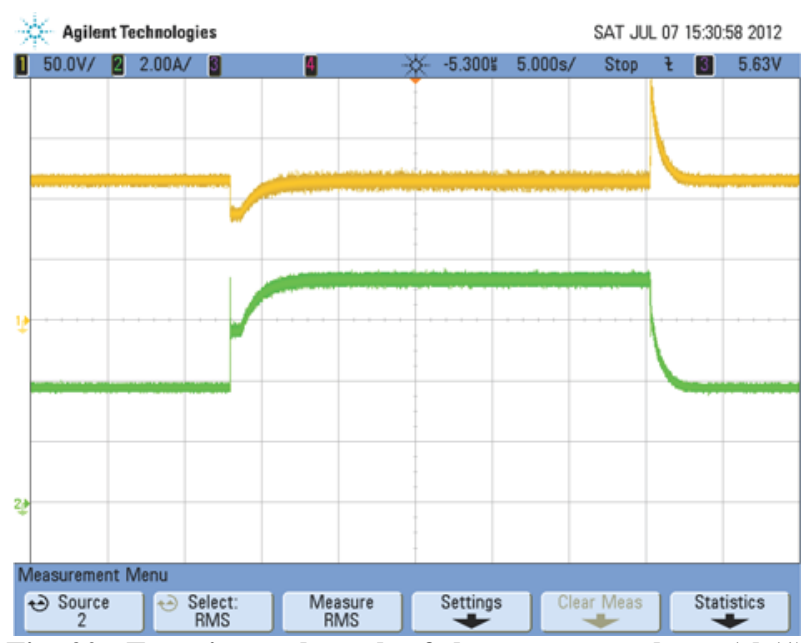

Fig. 20 Experimental result of the response voltage (ch(1) $50 \mathrm{~V} / \mathrm{div}$ ) and current (ch(2) 2A/div) for change load. 


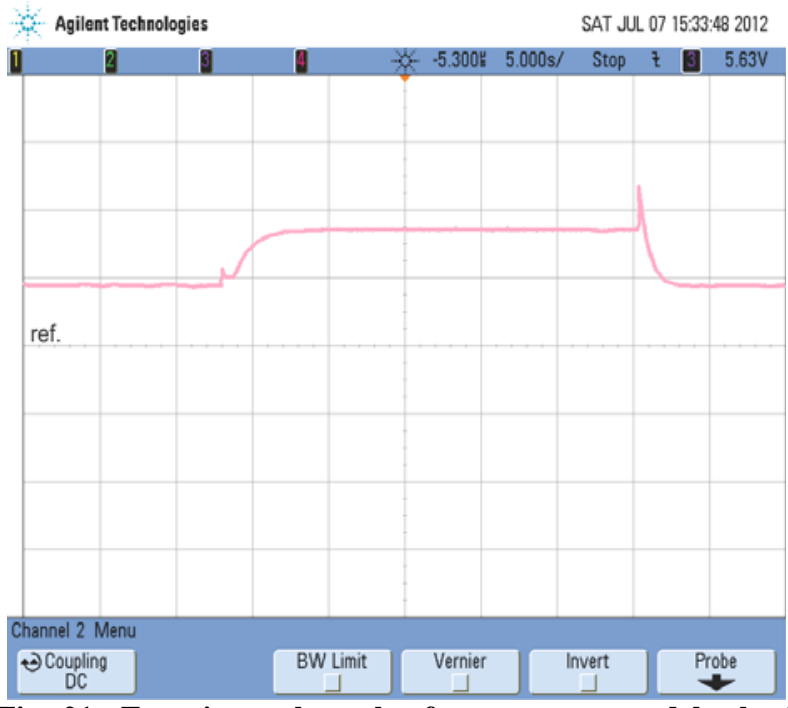

Fig. 21 Experimental result of power consumed by load (500 W/div).

It should be emphasized again that despite not predict the current peaks, leaving it to the more general model of the DAB, the methodology was effective for a rapid dimensioning.

\section{Acknowledgments}

The authors would like to thank the Brazilian agencies CNPq, CAPES and FINEP for the financial support, and INEP-Power Electronics Institute, by the technical support.

\section{References}

[1] M.H. Kheraluwala, D.W. Novotny, D.M. Divan, Design considerations for high power high frequency transformers, in: 21st Annual IEEE Power Electronics Specialists Conference, San Antonio, TX, USA, June 11-14, 1990, pp. 734-742.

[2] R.W.A.A. De Doncker, D.M. Divan, M.H. Kheraluwala, A three-phase soft-switched high-power-density DC/DC converter for high-power applications, IEEE Transactions on Industry Applications 27 (1) (1991) 63-73.

[3] B.D.H. Tellegen, The gyrator, a new electric network element, Philips Research Laboratories, the Netherlands, 1948.

[4] R.Y. Barazarte, X. Gonza, M. Ehsani, Generalized gyrator theory, IEEE Transactions on Power Electronics 25 (7) (2010) 1832-1837.

[5] M. Ehsani, I. Husain, M.O. Bilgic, Power converters as natural gyrators, circuits and systems, IEEE Transactions on Fundamental Theory and Applications 40 (12) (1993) 946-949.

[6] S. Singer, R.W. Erickson, Canonical modeling of power processing circuits based on the popi concept, IEEE Transactions on Power Electronics 7 (1) (1992) 37-43.

[7] C.D.M. Bilgic, Four-quadrant switch-mode gyrator, in: 18th European Conference on Circuit Theory and Design, Seville, Spain, 2007, pp. 1022-1025.

[8] A. Cid-Pastor, L. Martinez-Salamero, C. Alonso, G. Schweitz, Classification and synthesis of power gyrators, IEEE Proceedings Electric Power Applications 153 (6) (2006) 802-808. 